OPEN ACCESS

Edited by: Luis Puelles,

University of Murcia, Spain

Reviewed by:

Martin Wild

The University of Auckland,

New Zealand

Winfried Neuhuber,

University of Erlangen-Nuremberg,

Germany

*Correspondence:

Dominik Heyers

dominik.heyers@uni-oldenburg.de

Received: 12 January 2022 Accepted: 08 February 2022

Published: 07 March 2022

Citation:

Haase K, Musielak I,

Warmuth-Moles L, Leberecht B,

Zolotareva A, Mouritsen $\mathrm{H}$ and

Heyers D (2022) In Search

for the Avian Trigeminal Magnetic

Sensor: Distribution of Peripheral

and Central Terminals of Ophthalmic

Sensory Neurons

in the Night-Migratory Eurasian

Blackcap (Sylvia atricapilla).

Front. Neuroanat. 16:853401.

doi: 10.3389/fnana.2022.853401

\section{In Search for the Avian Trigeminal Magnetic Sensor: Distribution of Peripheral and Central Terminals of Ophthalmic Sensory Neurons in the Night-Migratory Eurasian Blackcap (Sylvia atricapilla)}

Katrin Haase ${ }^{1}$, Isabelle Musielak ${ }^{1}$, Leonie Warmuth-Moles ${ }^{1}$, Bo Leberecht ${ }^{1}$, Anna Zolotareva ${ }^{2}$, Henrik Mouritsen ${ }^{1,3}$ and Dominik Heyers ${ }^{1,3 *}$

${ }^{1}$ AG Neurosensorik, Institute of Biology and Environmental Sciences, Carl von Ossietzky Universität Oldenburg, Oldenburg, Germany, ${ }^{2}$ Biological Station Rybachy, Zoological Institute of Russian Academy of Sciences, St Petersburg, Russia,

${ }^{3}$ Research Centre for Neurosensory Sciences, Carl von Ossietzky Universität Oldenburg, Oldenburg, Germany

In night-migratory songbirds, neurobiological and behavioral evidence suggest the existence of a magnetic sense associated with the ophthalmic branch of the trigeminal nerve (V1), possibly providing magnetic positional information. Curiously, neither the unequivocal existence, structural nature, nor the exact location of any sensory structure has been revealed to date. Here, we used neuronal tract tracing to map both the innervation fields in the upper beak and the detailed trigeminal brainstem terminations of the medial and lateral V1 subbranches in the night-migratory Eurasian Blackcap (Sy/via atricapilla). The medial $\mathrm{V} 1$ subbranch takes its course along the ventral part of the upper beak to innervate subepidermal layers and the mucosa of the nasal cavity, whereas the lateral $\mathrm{V} 1$ subbranch runs along dorsolateral levels until the nostrils to innervate mainly the skin of the upper beak. In the trigeminal brainstem, medial V1 terminals innervate both the dorsal part and the ventral, magnetically activated part of the principal sensory trigeminal brainstem nuclei (PrV). In contrast, the lateral V1 subbranch innervates only a small part of the ventral PrV. The spinal sensory trigeminal brainstem nuclei (SpV) receive topographically ordered projections. The medial V1 subbranch mainly innervates rostral and medial parts of SpV, whereas the lateral V1 subbranch mainly innervates the lateral and caudal parts of SpV. The present findings could provide valuable information for further analysis of the trigeminal magnetic sense of birds.

Keywords: magnetoreception, neuroanatomy, somatosensory system, songbird, trigeminal system

\section{INTRODUCTION}

In birds, the perception of somatosensory information from the facial/beak region is of central importance for a wide range of behaviors. To mediate them, their underlying trigeminal sensory system has undergone a particularly high degree of diversification. In addition to mechanoreception, proprio-, thermo-, chemo-, and nociception, the birds' trigeminally mediated 
behavioral repertoire ranges from feeding, tactile exploration of the environment, hatching, grooming, climbing, and nest building (Wild, 2015; Faunes and Wild, 2017b) to cognitively complex skills such as skillful food handling (Ziswiler, 1965; Zweers et al., 1994) and/or the use of tools (Weir et al., 2002).

Trigeminal sensory information is transmitted via the fifth cranial, i.e., trigeminal nerve (N. V.), toward the brain. N. V. splits into ophthalmic (V1), maxillary (V2), and mandibular (V3) branches (Bubien-Waluszewska, 1981), all of which fuse in the trigeminal (Gasserian) ganglion to enter the brain at the level of the rostral pons. Toward the brain, N. V. splits into an ascending and a descending trigeminal tract to terminate in the principal $(\mathrm{PrV})$ and spinal $(\mathrm{SpV})$ sensory trigeminal brainstem nuclei, respectively (Wild and Zeigler, 1996). SpV, from rostral to caudal, can be subdivided into an oral, interpolar, and caudal part. $\mathrm{SpV}$ has projections within $\mathrm{SpV}$, and projects to $\mathrm{PrV}$ (Arends et al., 1984; Faunes and Wild, 2017a). PrV consists of an oval-shaped dorsal and a ventrally attached, crescentshaped part. These subparts relay trigeminal information to different parts of the forebrain (Wild et al., 1985; Wild and Farabaugh, 1996; Wild and Zeigler, 1996; Mouritsen et al., 2016; Kobylkov et al., 2020).

Some birds are also among nature's foremost navigators (Alerstam, 1993; Wiltschko and Wiltschko, 1995; Frost and Mouritsen, 2006; Mouritsen et al., 2016; Chernetsov, 2017; Chernetsov et al., 2017; Heyers et al., 2017; Mouritsen, 2018; Wynn et al., 2022). Accumulating evidence suggests that the trigeminal system, in particular $\mathrm{V} 1$, is also involved in the perception of geomagnetic information in night-migratory songbirds. Night-migratory songbirds, which can compensate for both geographical and virtual magnetic displacements by correcting their migratory direction (Chernetsov et al., 2008, 2017; Kishkinev et al., 2015, 2021), failed to do so when their V1s were surgically ablated. Instead, they headed in the same migratory direction as before the displacement (Kishkinev et al., 2013; Pakhomov et al., 2018). This indicates that V1 is involved in sensing magnetic positional information. On the brain level, the ventral part of $\mathrm{PrV}$ and the $\mathrm{SpV}$ were shown to display significantly increased neuronal activation when the birds were exposed to a strongly changing magnetic field stimulus. Both compensation of the ambient magnetic field or V1 ablation significantly decreased the number of magnetically activated neurons (Heyers et al., 2010; Lefeldt et al., 2014; Elbers et al., 2017). Unlike the dorsal part of PrV, which sends somatosensory information via the quintofrontal tract to the telencephalic nucleus basorostralis (Wild et al., 1985; Wild and Farabaugh, 1996; Wild and Zeigler, 1996; Kobylkov et al., 2020), the magnetically activated ventral part of $\mathrm{PrV}$ was recently shown to form the origin of a morphologically distinct neuronal population exclusively connected to the telencephalic frontal nidopallium. The ventral PrV part could thus represent part of a brain pathway specifically dedicated to transmitting magnetic map information to multisensory integration centers in the avian forebrain (Kobylkov et al., 2020).

In stark contrast to the growing body of evidence for V1-mediated magnetoreception and its central nervous representation, many attempts failed to find an underlying sensory structure (Williams and Wild, 2001; Fleissner et al., 2003, 2007; Falkenberg et al., 2010; Mouritsen, 2012; Treiber et al., 2012, 2013; Engels et al., 2018). Most of these studies used generic neuronal markers to label nerve-fiber terminals potentially harboring the magnetic sensor. One aspect which has only been sparsely investigated in this context is the detailed course of V1 up to its distal terminals in the upper beak. In fact, this has only been described in non-migratory chicken (Bubien-Waluszewska, 1981). Furthermore, while the V1 terminations in the trigeminal brainstem complex have been described in different species (Wild and Zeigler, 1996; Heyers et al., 2010; Lefeldt et al., 2014; Elbers et al., 2017; Faunes and Wild, 2017a,b; Kobylkov et al., 2020), these studies did not differentiate between terminations originating from different V1 subbranches, namely, the medial and lateral V1 subbranch. Assuming that any magnetic sensory structure should be located within or in the near vicinity of V1 fiber terminals, the specific mapping of the medial and lateral V1 subbranches in a nightmigratory songbird could narrow the regions to be searched for the elusive trigeminal magnetic sensors.

Therefore, the aim of the present study was to perform selective neuronal tract-tracing of the medial and lateral V1 subbranches in the long-distance night-migratory songbird Eurasian blackcap (Sylvia atricapilla) to map their respective courses from their distal nerve fiber terminals within the upper beak up to their projections in the trigeminal brainstem complex.

\section{MATERIALS AND METHODS}

\section{Animals}

A total of 8 male and 6 female adult Eurasian blackcaps (S. atricapilla) were used for this study. All animal procedures were approved by the Animal Care and Use Committees of the Niedersächsisches Landesamt für Verbraucherschutz und Lebensmittelsicherheit (LAVES, Oldenburg, Germany, Az.: 33.19-42502-04-15/1865; 33.19-42502-04-20/3492; 33.8-4250204-17/2724). The birds were wild-caught using mist nets after the breeding season and during autumn migration in the vicinity of University Oldenburg. The birds were housed in pairs in indoor wire cages $(102 \mathrm{~cm} \times 50 \mathrm{~cm} \times 40 \mathrm{~cm})$ at the institute's animal housing facility. They were kept at around $21^{\circ} \mathrm{C}$ and were exposed to a light regime simulating the natural circannual and circadian light-dark cycle of Oldenburg. Food and water were provided ad libitum.

\section{Neuronal Tract Tracing}

To visualize the course of $\mathrm{V} 1$ and its subbranches, neuronal tract tracer was either injected into the medial V1 subbranch, the lateral V1 subbranch, or the entire V1. Two different types of anesthesia were used to allow accessibility to the respective nerve subbranches: for entire V1 tracings, inhalation of anesthesia with $1-1.5 \%$ volume isoflurane $\mathrm{CP}^{\circledR}(1 \mathrm{ml} / \mathrm{ml}$; cp-pharma, Burgdorf, Germany) dissolved in oxygen and administered through a beak mask was used. For medial and lateral V1 tracings, we administered a mix of ketamine hydrochloride (Ketamin, 
WDT, Garbsen, Germany) and medetomidine (Domitor ${ }^{\circledR}$, Orion Pharma, Ismaning, Germany), each at a concentration of $0.1 \mathrm{ml} / \mathrm{kg}$ body weight dissolved in $0.9 \%$ sodium chloride $(\mathrm{NaCl})$, into the pectoral muscle. For the tracings, the animals were head-fixed. For entire V1 tracings, the nerve was accessed unilaterally through an incision along the dorsal rim of the orbit and careful retraction of the eyeball and oculomotor muscles (five animals). This procedure was identical to previous studies, in which V1 was either tracer-labeled or surgically sectioned (Zapka et al., 2009; Heyers et al., 2010; Kishkinev et al., 2013; Lefeldt et al., 2014; Elbers et al., 2017; Pakhomov et al., 2018). For medial V1 tracings, the subbranch was accessed through a small window cut at the inside of the upper beak at the caudal end of the cleft palate and nostrils (five animals). The lateral V1 subbranch was accessed through a windowcut at the lateral beak base (four animals). A total of 100$300 \mathrm{nl}$ of the neuronal tracer substance Cholera toxin B subunit (CtB; 1\% in distilled water; C9903, Sigma-Aldrich, St. Louis, MO, United States) was administered by pressure injections using a microinjector (WPI-2000, World Precision Instruments, Sarasota, FL, United States) and beveled glass capillaries. After the surgery, the skin and tissue were repositioned and resealed using cyanoacrylate surgical glue (Histoacryl ${ }^{\circledR}$, BRAUN, Rubi, Spain). Meloxicam (Metacam ${ }^{\circledR}$, Boehringer Ingelheim, Ingelheim, Germany; $0.1 \mathrm{ml} / \mathrm{kg}$ body weight dissolved in $0.9 \%$ $\mathrm{NaCl}$ ) was administered to each bird intramuscularly in the pectoral muscle after 24 and $48 \mathrm{~h}$ for post-surgical analgesia. The birds were given 5 days to recover from the surgery and to let the tracer be transported.

\section{Magnetic Stimulation}

To show which parts of the trigeminal brainstem complex are magnetically activated, birds were exposed to a magnetic field stimulus. Single birds were placed in a round Plexiglas cage on a wooden table at the center of a double-wrapped, three-axis Merrit four-coil system (Kirschvink, 1992). The coil system was housed in an aluminum-shielded chamber acting as a Faraday cage, which allowed static magnetic fields to pass through, while radiofrequency fields were attenuated (Engels et al., 2014; Schwarze et al., 2016). The three axes of the coil system were powered by three separate constant-current power supplies (BOP 50-4 M, Kepco Inc., Flushing, NY, United States), which were controlled by a custom-written MatLab script (Version: 2013a, Matlab, Mathworks, Natick, MS, United States; Lefeldt et al., 2014). The birds experienced a magnetic stimulus containing randomized small and large variations in two alternating 5min blocks: in the first $5 \mathrm{~min}$, only the horizontal direction of the magnetic field changed every $30 \mathrm{~s}$ by approximately $90^{\circ}$, while inclination (mean \pm standard deviation: $67.6 \pm 0.8^{\circ}$ ) and field intensity $(48,800 \pm 400 \mathrm{nT})$ only marginally changed. In the alternating $5 \mathrm{~min}$, the field intensity $(18,500-111,000 \mathrm{nT})$, horizontal direction $\left(0-360^{\circ}\right)$, and inclination $\left(-84.9 \pm 76.6^{\circ}\right)$ changed randomly and independently every $30 \mathrm{~s}$. This stimulus was identical to the one which was used previously and which consistently resulted in the activation of the trigeminal brainstem complex (Heyers et al., 2010; Lefeldt et al., 2014; Elbers et al., 2017; Kobylkov et al., 2020). The setup was illuminated with light bulbs at a light intensity of approximately $2.5 \mathrm{~mW} / \mathrm{m}^{2}$ (Zapka et al., 2009). As in previous studies, we tried to minimize any potential brain activation effects caused by excessive mechanical stimulation of the beak or motor activity (Feenders et al., 2008) by monitoring the behavior of each bird in real time using infrared cameras. A bird was only taken for brain analysis when it was constantly awake and sitting still during the magnetic exposure for at least 60-90 $\mathrm{min}$.

\section{Tissue Processing}

Immediately after magnetic exposure, birds were deeply anesthetized with pentobarbital $\left(\right.$ Narcoren $^{\circledR}$, Boehringer Ingelheim, Ingelheim, Germany; $2.5 \mathrm{ml} / \mathrm{kg}$ body weight) and transcardially perfused with $0.9 \% \mathrm{NaCl}$ followed by $4 \%$ paraformaldehyde (PFA) dissolved in phosphate-buffered saline (PBS; pH 7.4). Brains and beaks were extracted, post-fixed in $4 \%$ PFA in PBS for $24 \mathrm{~h}$, and cryoprotected in $30 \% \mathrm{D}(+)$-saccharose dissolved in PBS for at least $48 \mathrm{~h}$. Brains and beaks were cut using a freezing microtome (Leica CM 1860, Wetzlar, Germany). Brains were cut in $40-\mu \mathrm{m}$ thick slices in the frontal plane in six parallel series and the free-floating slices were collected in $0.1 \%$ sodium azide dissolved in PBS. Beaks were cut in $25-\mu$ m thick slices in the frontal plane in 10 parallel series, dried on gelatinized glass slides (Menzel SuperFrost ${ }^{\circledR}$ Plus, Thermo Fisher Scientific, Waltham, MA, United States), and stored at $-20^{\circ} \mathrm{C}$.

\section{Immunohistochemistry}

The permanent 3'3-diamino-benzidine (DAB) staining method was used to map the detailed course of the nerve (the basis of Figures 1, 2, 4) because this method is more sensitive and detects more fibers than immunofluorescence staining methods. For the double stainings (Figure 3); however, we had to use the less-sensitive fluorescence staining method.

For the permanent DAB staining, parallel series of brain or beak slices were stained together. Brain slices were stained freefloating, beak slices were stained on glass slides. Slices were washed three times for 10 min with Tris-buffered saline (TBS; $\mathrm{pH}$ 7.6). Endogenous peroxidases were saturated with $0.3 \%$ hydrogen peroxide for $30 \mathrm{~min}$. After three times of washing for 10 min each in TBS, unspecific binding sites were blocked with $10 \%$ normal donkey serum (NDS; Antibodies-online, Aachen, Germany) dissolved in TBS containing 0.3\% Triton-X100 (TBST; Carl Roth, Karlsruhe, Germany) for $30 \mathrm{~min}$. Slices were incubated either with a primary polyclonal rabbit anti-CtB antibody (1:1000 in 5\% NDS in TBS-T; C3062, lot. 045M4864V, Sigma-Aldrich, St. Louis, MO, United States, RRID: AB_258833) overnight at $4^{\circ} \mathrm{C}$, with the general neuronal marker monoclonal mouse anti-HuC/HuD antibody (1:500 in 5\% NDS in TBS-T; A21272, lot. 1963099, Invitrogen, Carlsbad, CA, United States, RRID: AB_2535822) overnight at $4^{\circ} \mathrm{C}$, or with a polyclonal rabbit anti-Egr-1 antibody (1:1000 in 5\% NDS in TBS-T; SC-189, lot. A2516, Santa Cruz Biotechnology, Dallas, TX, United States, RRID: AB_2231020) for $72 \mathrm{~h}$ at $4^{\circ} \mathrm{C}$.

After washing three times for $10 \mathrm{~min}$ each in TBS, slices were incubated with a biotinylated secondary antibody (1:500 for brain slices, 1:200 for beak slices in TBS-T; PK-4002, PK-6101, Vector 


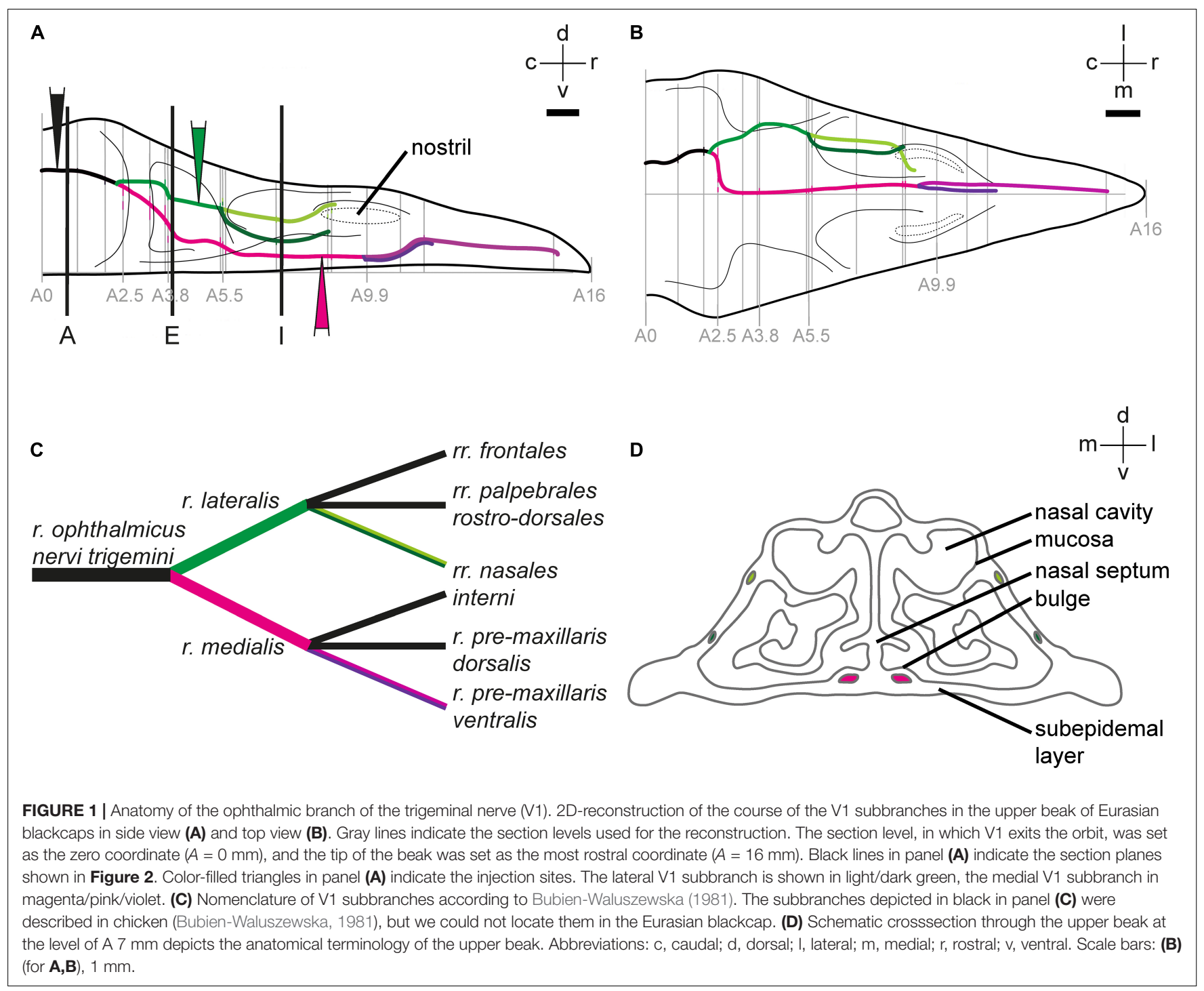

Laboratories, Burlingame, CA, United States) for $120 \mathrm{~min}$ at room temperature. After three washing steps for $10 \mathrm{~min}$ each in TBS-T, the slices were incubated in an avidin-coupled peroxidase complex (according to the manufacturer's instructions, PK-4002, PK-6101, Vector Laboratories, Burlingame, CA, United States) for $60 \mathrm{~min}$ at room temperature. After two washing steps of $10 \mathrm{~min}$ each in TBS and inactivation of ongoing reactions in $0.1 \mathrm{M}$ sodium acetate for $5 \mathrm{~min}$, peroxidase activity was visualized under continuous visual inspection for $20 \mathrm{~min}$ using a 3'3-diamino-benzidine (Acros Organics, Fair Lawn, NJ, United States) reaction using glucose oxidase $(0.2 \%$ in glycerol and distilled water; Sigma-Aldrich, St. Louis, MO, United States) instead of hydrogen peroxide (Shu et al., 1988). The reaction was stopped by incubating the slices in $0.1 \mathrm{M}$ sodium acetate for $5 \mathrm{~min}$. The stained brain slices were mounted on glass slides (Menzel SuperFrost ${ }^{\circledR}$ Plus, Thermo Fisher Scientific, Waltham, MA, United States). Brain and beak slices were dehydrated in a graded series of alcohol (70\% ethanol, 96\% ethanol, isopropanol, twice xylene) and cover-slipped with Eukitt
(Sigma-Aldrich, St. Louis, MO, United States). Negative controls were performed on parallel beak slices omitting the primary antibody to distinguish endogenous pigment epithelium from the substrate staining. Brain and beak slices were imaged using light microscopy (Zeiss Axio Scan.Z1, Oberkochen, Germany, objective: $10 \times$ Plan-Apochromat, 0.45 NA). Contrast was adjusted with identical settings using the function Enhance contrast of Image (Version: 1.53f51, NIH, Bethesda, MD, United States; RRID: SCR_003070; Schindelin et al., 2012).

For fluorescent immunohistochemical stainings, beak slices were washed three times in TBS for $10 \mathrm{~min}$ each, and unspecific binding sites were blocked with 10\% NDS (in TBS-T; Antibodiesonline, Aachen, Germany) for $1 \mathrm{~h}$. Slices were incubated with a polyclonal rabbit anti-CtB antibody (1:1000 in 5\% NDS in TBS-T; C3062, lot. 045M4864V, Sigma-Aldrich, St. Louis, MO, United States, RRID: AB_258833) and a monoclonal mouse anti-Tubulin beta 3 (TUBB3) antibody (1:500 in 5\% NDS in TBS-T; 801201, lot. B249869, BioLegend, San Diego, CA, United States, RRID: AB_10063408) overnight at $4^{\circ} \mathrm{C}$. Slices 


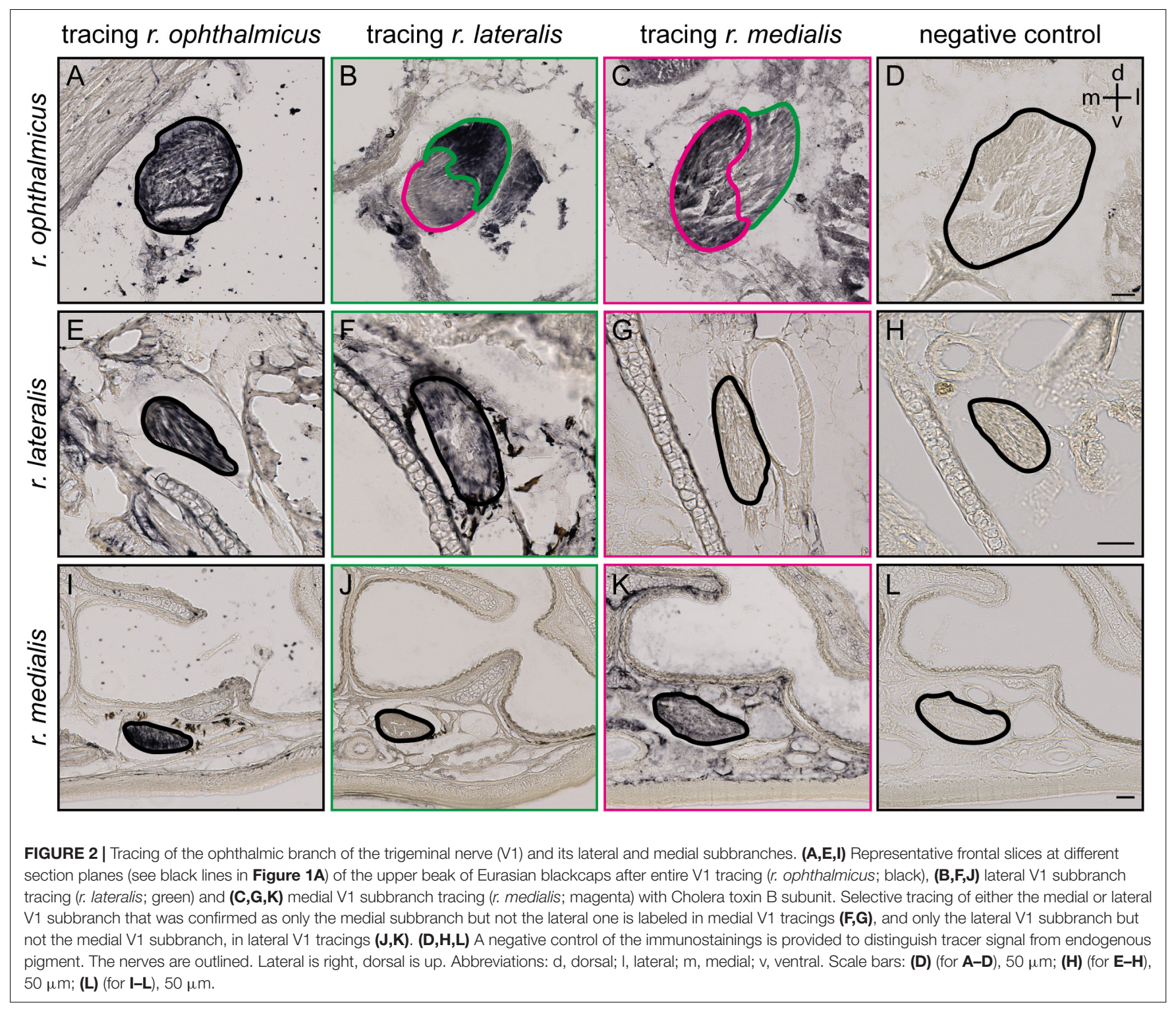

were washed three times 5 min each in TBS before the TUBB3 signal was enhanced by incubating the slices with a secondary biotinylated horse anti-mouse antibody (1:500 in TBS-T; PK4002, Vector Laboratories, Burlingame, CA, United States) for $90 \mathrm{~min}$ at room temperature. After three times of 5-min washing each in TBS, primary antibodies were detected with Alexa-conjugated secondary antibodies (each 1:500 in TBS-T; Streptavidin Alexa Fluor 488 conjugate, S32354, Invitrogen, Carlsbad, CA, United States; Alexa Fluor 568 donkey antirabbit, ab175692, Abcam, Cambridge, United Kingdom) on incubation for $90 \mathrm{~min}$ at room temperature. Slices were coverslipped with 4',6-Diamidin-2-phenylindol ( $2 \mu \mathrm{g} / \mathrm{ml}$, Carl Roth, Karlsruhe, Germany) dissolved in DABCO (Carl Roth, Karlsruhe, Germany) and Mowiol (Carl Roth, Karlsruhe, Germany). Negative controls were performed on parallel beak slices omitting the primary antibodies. Beak slices were imaged using fluorescence microscopy (Leica DM6 B, Wetzlar, Germany, objective: $20 \times$ HC PL FLUOTAR, 0.50 NA). With ImageJ (Version: 1.53f51, NIH, Bethesda, MD, United States; RRID: SCR_003070; Schindelin et al., 2012) background subtraction was done, the contrast was adjusted using the function Enhance contrast, and the maximum projection of each stack was used, all with identical settings.

\section{Anatomical Mapping}

To reconstruct the course of $\mathrm{V} 1$ and its subbranches, a series of beak slices stained against CtB were systematically aligned and normalized based on hand drawings in a coordinate grid using a pen display (three animals; Wacom Cintiq 21UX, Wacom, Düsseldorf, Germany) and the Adobe Illustrator 25.4.1 software (Adobe Systems Software, Dublin, Ireland, RRID: SCR_010279). The course of V1 and its subbranches was translated into 2D reconstructions, one depicting the course of the subbranches as a side view (Figure 1A) showing the dorsoventral coordinates of 


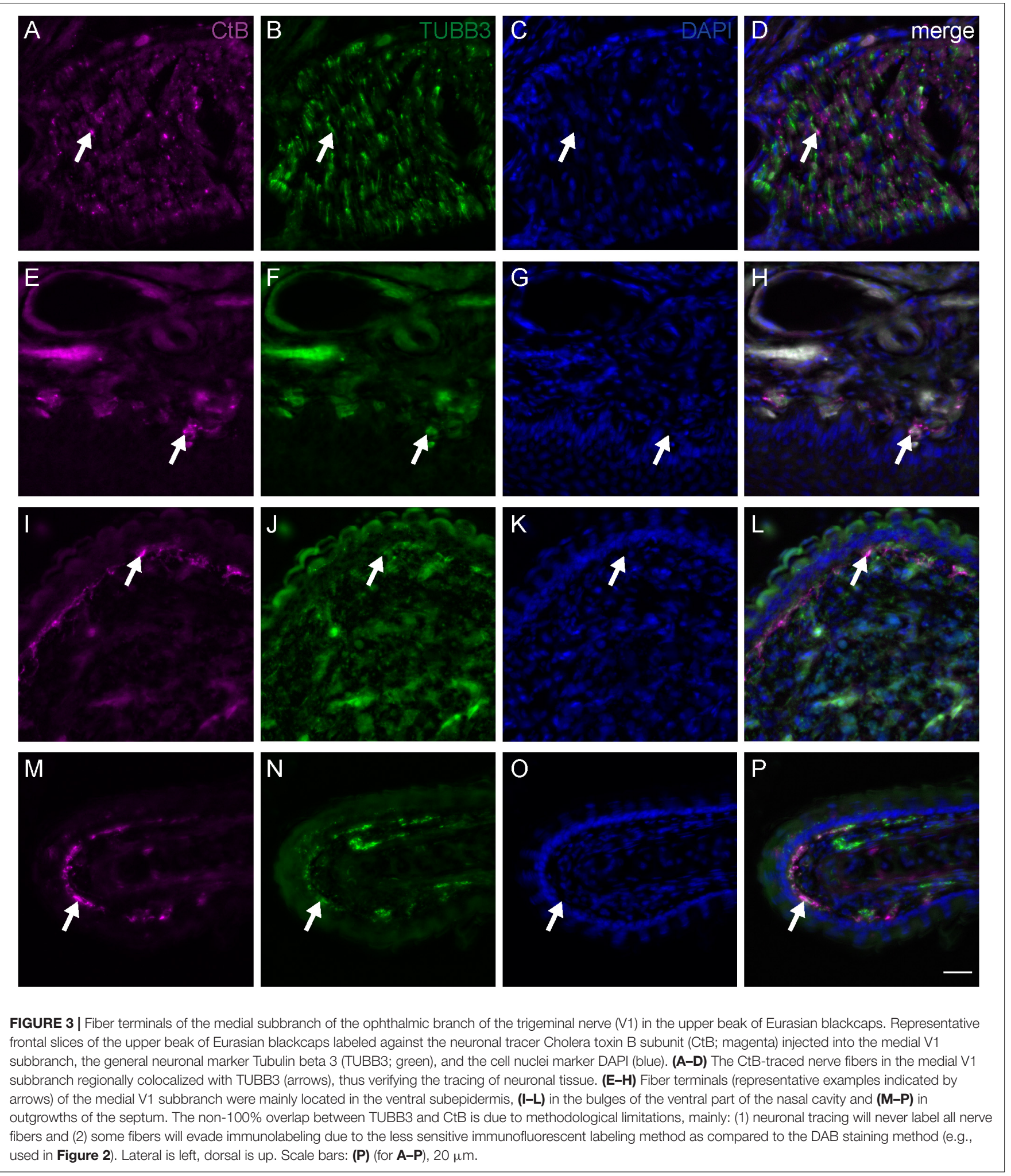

the nerve subbranches, and one as viewed from above (Figure 1B) to show the mediolateral coordinates. Anatomical boundaries within the trigeminal brainstem complex were defined based on previous studies on the known restricted expression of the immediate early gene Egr-1 and on morphometric features using the general neuronal marker $\mathrm{HuC} / \mathrm{HuD}$ (Heyers et al., 2010; Lefeldt et al., 2014; Elbers et al., 2017; Faunes and Wild, 2017b; Kobylkov et al., 2020). 



\section{RESULTS}

\section{Methodological Considerations}

During the experiments, we took the greatest care to make our tracings as replicable as possible, i.e., by accessing the nerve subbranches at the same location within the beak and injecting the same amounts of tracer. Nevertheless, due to the predictable methodological limitations of this technique, we observed slightly different innervation patterns between single specimens. However, all our core findings were observable in all cases, and for the figures, we carefully compared all "cases" and chose the most representative ones to appear in our manuscript.

\section{V1 Subbranch Course in the Upper Beak}

To identify the exact course and the distal terminations of V1 within the upper beak, we either selectively traced the medial or the lateral V1 subbranch in the night-migratory songbird Eurasian blackcap (Figures 1A, 2B,C,F,G,J,K; magenta and green). For comparison, we traced the entire V1 at a level where the medial and lateral V1 subbranch had already fused into one nerve (Figures 1A, 2A,E,I; black). We confirmed the successful selective tracing by analyzing the tracing patterns in beak slices. At the caudal end of the upper beak beyond the point where the medial and lateral V1 subbranches are fused, only selective V1 parts were labeled by medial or lateral $\mathrm{V} 1$ tracings (Figures 2A-D). In rostral parts of the upper beak either only the medial V1 subbranch was labeled by V1 medial tracings or the lateral V1 subbranch by lateral V1 tracings (Figures 2E-L). Furthermore, double immunofluorescence stainings confirmed that we indeed traced neuronal tissue, depicted as a partial colocalization of the tracer $\mathrm{CtB}$ (magenta) and the neurofilament marker TUBB3 (green; Figures 3A-D). The section level, in which V1 exits the orbit, was set as the anterior-posterior zero coordinate (approximately A $0 \mathrm{~mm}$ ), and the tip of the beak was set as the dorso-ventral zero coordinate (approximately A $16 \mathrm{~mm}$ ).

The ophthalmic branch of the trigeminal nerve leaves the orbit rostrally through the ophthalmic foramen, medially accompanied by the olfactory nerve (Figures 1A,B, 2A-D). V1 fuses with parts of the conchal lobes, and at approximately A $2.5 \mathrm{~mm}$ it rostrally bifurcates into its medial ( $r$. medialis; magenta) and lateral subbranches ( $r$. lateralis; green; Figures 1A-C).

The medial V1 subbranch turns ventromedial to find its way through the nasal septum and reaches the base of the upper beak at approximately A $3.8 \mathrm{~mm}$. On its way toward the tip of the upper beak, it separates into two further subbranches approximately at the level of the nostrils (A $9.9 \mathrm{~mm}$; Figures 1A-C, pink and violet). These subbranches likely represent the $r$. pre-maxillaris ventralis, the main continuation of the $r$. medialis (Figures 1C, 2I-L). Both, r. pre-maxillaris ventralis subbranches run in parallel further rostrally. Its larger portion runs laterally (pink) and can be tracked almost up to the tip of the beak (approximately A $15.7 \mathrm{~mm}$; Figures 1A-C). The $r$. pre-maxillaris ventralis innervates the mucosa of the rostral half of the palate and the tip of the upper beak (Bubien-Waluszewska, 1981). Distal fiber terminals of the medial V1 subbranch are mainly located in the ventral subepidermis (Figures $3 \mathbf{E}-\mathbf{H}$ ), in the bulges of the ventral part of the nasal cavity (Figures 3I-L), and in outgrowths of the septum (Figures 3M-P; see Figure 1D for anatomical terminology). The rr. nasales interni and the $r$. premaxillaris dosalis of the $r$. medials described in chicken (BubienWaluszewska, 1981) could not be found in blackcaps (Figure 1C).

After separating from the medial V1 subbranch, the lateral V1 subbranch runs rostrally at approximately the same dorso-ventral level as the entire V1. At approximately A $5.5 \mathrm{~mm}$, it splits up into two further subbranches, likely resembling the rr. nasales interni [Figures 1A-D; light and dark green; very similar to what was shown in chicken (Bubien-Waluszewska, 1981)]. Both initially take their course inside the nasal bone, while the dorsal subbranch (light green) exits the nasal bone again and takes its course on its surface (Figures 1A,B, 2E-H). Both can be tracked until the opening of the nostrils become apparent (at A $9.9 \mathrm{~mm}$; Figures 1A,B). The rr. nasales interni are known to innervate the mucosa of the lateral nasal cavity (Bubien-Waluszewska, 1981). Two further subbranches known from chicken (BubienWaluszewska, 1981), i.e., the $r$. frontales and the $r r$. palpebrales rostro-dorsales, could not be identified in blackcaps (Figure 1C).

\section{V1 Subbranch Projections to the Trigeminal Brainstem Complex}

To identify the trigeminal brainstem terminations of the medial and lateral V1 subbranches, we selectively injected neuronal tracer into the respective subbranches (Figures 1A, 4E-L; magenta and green) and analyzed the anterograde innervation patterns in the PrV and SpV in Eurasian blackcaps. Additionally, neuronal tracings of the entire V1 were performed for comparison of the innervation patterns (Figures 4A-D; black). To define the neuroanatomical boundaries of the respective brain areas, a general neuronal marker $(\mathrm{HuC} / \mathrm{HuD})$ was used (Figures $4 \mathbf{M}-\mathbf{P}$ ). The dorsal and ventral parts of $\mathrm{PrV}$ were previously shown to be distinguishable based on their respective soma sizes (Figure 4M; Kobylkov et al., 2020).

Medial subbranch tracings revealed terminations in both the medial and lateral parts of the dorsal part of PrV and a termination field in the magnetically activated ventral part of PrV (Figures 4E,U; magenta), depicted by the expression of the immediate early gene Egr-1 on parallel brain slices (Figures 4Q,U). Lateral V1 subbranch tracings revealed a small region with fiber terminals in the ventral tip of $\mathrm{PrV}$ (Figures 4I,U; green). The termination field of the medial and lateral V1 subbranches partially overlapped in the ventral part of $\operatorname{PrV}$ (Figure 4U).

In the $\mathrm{SpV}$, medial $\mathrm{V} 1$ subbranch tracings resulted in the labeling of fiber terminals mainly in all medial parts of $\mathrm{SpV}$ and in small parts of the lateral part of $\mathrm{SpV}$ (Figures $4 \mathbf{F}-\mathbf{H}, \mathbf{V}-\mathbf{X}$; magenta). Lateral V1 subbranch tracings labeled fiber terminals mainly in lateral parts of $\mathrm{SpV}$. However, the termination field gradually moved medial toward caudal $\mathrm{SpV}$ levels (Figures 4J-L,V-X; green). The termination fields of the medial and lateral V1 subbranches in SpV partially overlapped, while generally conserving the topographic innervation (Figures 4V-X). 


\section{DISCUSSION}

Previous neurobiological studies have suggested an involvement of the trigeminal brainstem complex in processing magnetic information. More specifically, the ventral part of $\operatorname{PrV}$ and parts of $\mathrm{SpV}$ were shown to display magnetic field-induced, V1-mediated, neuronal activation (Heyers et al., 2010; Lefeldt et al., 2014; Elbers et al., 2017). Recently, we identified a previously unknown brain pathway, with which magnetic information from the ventral part of $\mathrm{PrV}$ is most likely being sent to the telencephalic frontal nidopallium (Kobylkov et al., 2020). Furthermore, physical, geographical, and virtual magnetic displacements have indicated that V1, which innervates the trigeminal brainstem complex, is essential for providing magnetic positional information (Kishkinev et al., 2013; Pakhomov et al., 2018).

All aforementioned studies mapped V1 connectivities and hypothesized on its potential functions based on tracings and ablations of the entire V1. In contrast, this study provides the first precise neuroanatomical data from a night-migratory songbird on the exact course of V1 down to the level of its subbranches and their distal terminals and its proximal terminations in the trigeminal brainstem complex. The dorsal part of $\operatorname{PrV}$, known to be involved in processing somatosensory information, is exclusively innervated by the medial V1 subbranch (Figures $\mathbf{4 E}, \mathbf{U}$ ). The magnetically activated ventral part of $\operatorname{PrV}$ receives input from both the medial and lateral V1 subbranches (Figures 4E,I,Q,U). In SpV, the topographic innervation is mostly conserved, i.e., with the majority of fibers terminating in the medial parts belonging to the medial V1 subbranch, while the lateral V1 subbranch mainly terminates in lateral parts of SpV (Figures 4F-H,J-L, V-X).

In addition, we mapped the detailed course of each of the V1 subbranches up to its distal fiber terminals in the upper beak of a night-migratory songbird. The medial V1 subbranch runs along the ventral part of the upper beak to innervate its subepidermal layers and the septal and ventral parts of the mucosa within the nasal cavity (Figures 1, 2, 3). The lateral V1 subbranch runs along the dorso-lateral part of the beak innervating superficial tissue such as the skin of the forehead, the upper eyelid, the conjunctiva of the nasal commissure, and major parts of the mucosa of the nasal cavity (Figures 1, 2; Bubien-Waluszewska, 1981). Comparing the currently available literature with the respective courses of the tracer-labeled subbranches, our findings generally reflect the described anatomy of $\mathrm{V} 1$ and its subbranches in chicken (Bubien-Waluszewska, 1981), suggesting that the neuroanatomy of $\mathrm{V} 1$ is mainly conserved across the avian clade.

Do the restricted innervation patterns allow us to assign processing of information of a certain quality (somatosensation, magnetoreception, and/or both) to each of the V1 subbranches? Our data indicate that the medial V1 is most likely responsible for mediating somatosensory information from the upper beak since it is the only V1 subbranch that exclusively innervates the dorsal part of $\operatorname{PrV}$ (Figures 4E,U). The dorsal part of $\operatorname{PrV}$ is known to form the origin of fibers carrying somatosensory information (mechanoreception, proprio-, thermo-, chemo-, and nociception) to the telencephalic Nucleus basorostralis
(Wild et al., 1985; Kobylkov et al., 2020). This fact is supported by the location of medial V1 fiber terminals in the ventral subepidermal layer of the upper beak, the septum, and ventral parts of the nasal cavity (Figures 3E-P). These beak parts contain a dense multisensory innervation network, which birds will almost certainly consult when performing somatosensorymediated behaviors with their beaks such as feeding, grooming, climbing, nest building, hatching, and exploring the environment (Wild, 2015). The fact that additional medial V1 fibers terminate in the magnetically ventral part of $\operatorname{PrV}$ and that many medial V1 fibers terminate in magnetically activated parts of $\mathrm{SpV}$ (Figures $4 \mathrm{E}-\mathrm{L}, \mathrm{Q}-\mathrm{X}$ ) suggests that the medial V1 subbranch could also be involved in the processing of magnetic information. The lateral V1 subbranch solely terminates in a small portion of the magnetically activated ventral part of $\operatorname{PrV}$ (Figures 4I,Q,U), which would point toward a potential primary involvement in the processing of magnetic information. However, in the SpV, only some parts of the lateral V1 fibers terminate in magnetically activated $\mathrm{SpV}$ portions (Figures $\mathbf{4 J}-\mathbf{L}, \mathbf{R}-\mathbf{T}, \mathbf{V}-\mathbf{X}$ ), which would mean that some but not all lateral V1 fibers could be also involved in magnetoreception. Furthermore, some lateral subbranch neurons could still be involved in conveying somatosensory information to the dorsal part of PrV via interneurons passing on information from $\mathrm{SpV}$ to PrV.

Based on our expectation that any magnetic sensor must be located within the proximity of any of the V1 subbranches, do our data allow us to narrow in on the exact location of any magnetic sensor? At present, the answer is no since our data show that both subbranches innervate different magnetically activated parts of the trigeminal brainstem complex to varying degrees (Figure 4). Thus, based on our findings, it seems reasonable that both the medial as well as the lateral V1 subbranch might be involved in mediating magnetic information. Apart from the fact that assigning a specific function to a specific nerve subbranch is certainly too simplistic, any functional analyses based on purely anatomical data in our study are further hampered by the simple fact that (1) the lateral V1 subbranch is smaller than its medial counterpart (Figure 2), i.e., it consists of fewer neurons and, consequently, has both a smaller distal and proximal dendritic innervation field, (2) the magnetically activated ventral part of $\mathrm{PrV}$ does seem to contain a mix of neurons encoding for both magnetic and somatosensory information, since neuronal tract tracings from the connected telencephalic frontal nidopallium labeled only $15 \%$ of the magnetically activated neurons in the ventral part of $\operatorname{PrV}$ (Kobylkov et al., 2020), and, (3) we cannot rule out any possible interneuronal connectivities within the respective trigeminal brainstem subcompartments.

Only a highly elaborate correlation to function approach, e.g., the analysis of magnetic field-induced neuronal activation and/or a behavioral displacement study after selective ablation of either the medial or lateral V1 subbranch could give further evidence about any specific involvement of one of the V1 subbranches in magnetoreception. However, given the complicated innervation patterns seen here, the estimated number of experimental animals needed to reach a clear conclusion, if any exists that could be obtained from such studies, would exceed our ethical limits, and we have therefore chosen not to perform such studies. 
In conclusion, the elusive trigeminal-based magnetic sensors could be located anywhere along the entire dendritic field of both V1 subbranches.

\section{DATA AVAILABILITY STATEMENT}

The original contributions presented in the study are included in the article/supplementary material, further inquiries can be directed to the corresponding author.

\section{ETHICS STATEMENT}

The animal study was reviewed and approved by the Animal Care and Use Committees of the Niedersächsisches Landesamt für Verbraucherschutz und Lebensmittelsicherheit (LAVES, Oldenburg, Germany, Az.: 33.19-42502-04-15/1865; 33.1942502-04-20/3492; and 33.8-42502-04-17/2724).

\section{AUTHOR CONTRIBUTIONS}

$\mathrm{DH}$ and HM designed the research. KH, IM, LW-M, BL, AZ, and $\mathrm{DH}$ performed experiments. $\mathrm{KH}, \mathrm{IM}, \mathrm{LW}-\mathrm{M}$, and $\mathrm{DH}$ analyzed the data. HM provided facilities. $\mathrm{KH}$ and $\mathrm{DH}$ wrote the first draft of the manuscript, which all co-authors commented

\section{REFERENCES}

Alerstam, T. (1993). Bird Migration. Cambridge: Cambridge University Press.

Arends, J. J., Woelders-Blok, A., and Dubbeldam, J. L. (1984). The efferent connections of the nuclei of the descending trigeminal tract in the mallard (Anas platyrhynchos L.). Neuroscience 13, 797-817. doi: 10.1016/0306-4522(84) 90097-6

Bubien-Waluszewska, A. (1981). "The cranial nerves," in Form and Function in Birds, eds A. S. King and J. McLelland (New York, NY: Academic Press), 385-438.

Chernetsov, N. (2017). Compass systems. J. Comp. Physiol. A 203, 447-453. doi: 10.1007/s00359-016-1140-x

Chernetsov, N., Kishkinev, D., and Mouritsen, H. (2008). A long-distance avian migrant compensates for longitudinal displacement during spring migration. Curr. Biol. 18, 188-190. doi: 10.1016/j.cub.2008.01.018

Chernetsov, N., Pakhomov, A., Kobylkov, D., Kishkinev, D., Holland, R. A., and Mouritsen, H. (2017). Migratory Eurasian reed warblers can use magnetic declination to solve the longitude problem. Curr. Biol. 27, 2647-2651.e2642. doi: 10.1016/j.cub.2017.07.024

Elbers, D., Bulte, M., Bairlein, F., Mouritsen, H., and Heyers, D. (2017). Magnetic activation in the brain of the migratory northern wheatear (Oenanthe oenanthe). J. Comp. Physiol. A 203, 591-600. doi: 10.1007/s00359-017-1 167-7

Engels, S., Schneider, N. L., Lefeldt, N., Hein, C.-M., Zapka, M., Michalik, A., et al. (2014). Anthropogenic electromagnetic noise disrupts magnetic compass orientation in a migratory bird. Nature 509, 353-356. doi: 10.1038/nature1 3290

Engels, S., Treiber, C. D., Salzer, M. C., Michalik, A., Ushakova, L., Keays, D. A., et al. (2018). Lidocaine is a nocebo treatment for trigeminally mediated magnetic orientation in birds. J. R. Soc. Interface 15:20180124. doi: 10.1098/rsif. 2018.0124

Falkenberg, G., Fleissner, G., Schuchardt, K., Kuehbacher, M., Thalau, P., Mouritsen, H., et al. (2010). Avian magnetoreception: elaborate iron mineral containing dendrites in the upper beak seem to be a common feature of birds. PLoS One 5:e9231. doi: 10.1371/journal.pone.0009231 on. All authors contributed to the article and approved the submitted version.

\section{FUNDING}

Our research was generously funded by the Deutsche Forschungsgemeinschaft (DFG; project number: 395940726-SFB 1372 "Magnetoreception and Navigation in Vertebrates" to DH and HM employing KH, IM, and BL; GRK 1885 "Molecular basis of sensory biology" to HM), the German Academic Exchange Service (DAAD; Leonard-Euler stipend to AZ and $\mathrm{DH}$ ), the Zoological Institute of Russian Academy of Sciences (research project 1021051603198-5 to AZ), and the European Research Council [under the European Union's Horizon 2020 Research and Innovation Program, grant agreement no. 810002 (Synergy Grant: "QuantumBirds" to HM)].

\section{ACKNOWLEDGMENTS}

We gratefully acknowledge the University's animal keeping facility for taking care of the birds and the University's Fluorescence Microscopy Service Unit for providing and maintaining imaging facilities. We also thank Patrick Dömer for microscopic support.

Faunes, M., and Wild, J. M. (2017b). The sensory trigeminal complex and the organization of its primary afferents in the zebra finch (Taeniopygia guttata). J. Comp. Neurol. 525, 2820-2831. doi: 10.1002/cne.24249

Faunes, M., and Wild, J. M. (2017a). The ascending projections of the nuclei of the descending trigeminal tract (nTTD) in the zebra finch (Taeniopygia guttata). J. Comp. Neurol. 525, 2832-2846. doi: 10.1002/cne.24247

Feenders, G., Liedvogel, M., Rivas, M., Zapka, M., Horita, H., Hara, E., et al. (2008). Molecular mapping of movement-associated areas in the avian brain: a motor theory for vocal learning origin. PLoS One 3:e1768. doi: 10.1371/journal.pone. 0001768

Fleissner, G., Holtkamp-Rötzler, E., Hanzlik, M., Winklhofer, M., Fleissner, G., Petersen, N., et al. (2003). Ultrastructural analysis of a putative magnetoreceptor in the beak of homing pigeons. J. Comp. Neurol. 458, 350-360. doi: 10.1002/cne. 10579

Fleissner, G., Stahl, B., Thalau, P., Falkenberg, G., and Fleissner, G. (2007). A novel concept of Fe-mineral-based magnetoreception: histological and physicochemical data from the upper beak of homing pigeons. Naturwissenschaften 94, 631-642. doi: 10.1007/s00114-007-0236-0

Frost, B. J., and Mouritsen, H. (2006). The neural mechanisms of long distance animal navigation. Curr. Opin. Neurobiol. 16, 481-488. doi: 10.1016/j.conb. 2006.06.005

Heyers, D., Elbers, D., Bulte, M., Bairlein, F., and Mouritsen, H. (2017). The magnetic map sense and its use in fine-tuning the migration programme of birds. J. Comp. Physiol. A 203, 491-497. doi: 10.1007/s00359-017-1 164-x

Heyers, D., Zapka, M., Hoffmeister, M., Wild, J. M., and Mouritsen, H. (2010). Magnetic field changes activate the trigeminal brainstem complex in a migratory bird. Proc. Natl. Acad. Sci. U. S. A. 107, 9394-9399. doi: 10.1073/ pnas.0907068107

Kirschvink, J. L. (1992). Uniform magnetic fields and double-wrapped coil systems: improved techniques for the design of bioelectromagnetic experiments. Bioelectromagnetics 13, 401-411. doi: 10.1002/bem.2250130507

Kishkinev, D., Chernetsov, N., Heyers, D., and Mouritsen, H. (2013). Migratory reed warblers need intact trigeminal nerves to correct for a $1,000 \mathrm{~km}$ eastward displacement. PLoS One 8:e65847. doi: 10.1371/journal.pone.0065847 
Kishkinev, D., Chernetsov, N., Pakhomov, A., Heyers, D., and Mouritsen, H. (2015). Eurasian reed warblers compensate for virtual magnetic displacement. Curr. Biol. 25, R822-R824. doi: 10.1016/j.cub.2015.08.012

Kishkinev, D., Packmor, F., Zechmeister, T., Winkler, H.-C., Chernetsov, N., Mouritsen, H., et al. (2021). Navigation by extrapolation of geomagnetic cues in a migratory songbird. Curr. Biol. 31, 1563-1569.e1564. doi: 10.1016/j.cub. 2021.01.051

Kobylkov, D., Schwarze, S., Michalik, B., Winklhofer, M., Mouritsen, H., and Heyers, D. (2020). A newly identified trigeminal brain pathway in a nightmigratory bird could be dedicated to transmitting magnetic map information. Proc. Biol. Sci. 287:20192788. doi: 10.1098/rspb.2019.2788

Lefeldt, N., Heyers, D., Schneider, N. L., Engels, S., Elbers, D., and Mouritsen, H. (2014). Magnetic field-driven induction of ZENK in the trigeminal system of pigeons (Columba livia). J. R. Soc. Interface 11:20140777. doi: 10.1098/rsif.2014. 0777

Mouritsen, H. (2012). Sensory biology: search for the compass needles. Nature 484, 320-321. doi: 10.1038/484320a

Mouritsen, H. (2018). Long-distance navigation and magnetoreception in migratory animals. Nature 558, 50-59. doi: 10.1038/s41586-018-0176- 1

Mouritsen, H., Heyers, D., and Güntürkün, O. (2016). The neural basis of longdistance navigation in birds. Annu. Rev. Physiol. 78, 133-154. doi: 10.1146/ annurev-physiol-021115-105054

Pakhomov, A., Anashina, A., Heyers, D., Kobylkov, D., Mouritsen, H., and Chernetsov, N. (2018). Magnetic map navigation in a migratory songbird requires trigeminal input. Sci. Rep. 8:11975. doi: 10.1038/s41598-018-30477-8

Schindelin, J., Arganda-Carreras, I., Frise, E., Kaynig, V., Longair, M., Pietzsch, T., et al. (2012). Fiji: an open-source platform for biological-image analysis. Nat. Methods 9, 676-682. doi: 10.1038/nmeth.2019

Schwarze, S., Steenken, F., Thiele, N., Kobylkov, D., Lefeldt, N., Dreyer, D., et al. (2016). Migratory blackcaps can use their magnetic compass at 5 degrees inclination, but are completely random at 0 degrees inclination. Sci. Rep. 6:33805. doi: $10.1038 /$ srep33805

Shu, S., Ju, G., and Fan, L. (1988). The glucose oxidase-DAB-nickel method in peroxidase histochemistry of the nervous system. Neurosci. Lett. 85, 169-171. doi: 10.1016/0304-3940(88)90346-1

Treiber, C. D., Salzer, M., Breuss, M., Ushakova, L., Lauwers, M., Edelman, N., et al. (2013). High resolution anatomical mapping confirms the absence of a magnetic sense system in the rostral upper beak of pigeons. Commun. Integr. Biol. 6:e24859. doi: 10.4161/cib.24859

Treiber, C. D., Salzer, M. C., Riegler, J., Edelman, N., Sugar, C., Breuss, M., et al. (2012). Clusters of iron-rich cells in the upper beak of pigeons are macrophages not magnetosensitive neurons. Nature 484, 367-370. doi: 10.1038/nature11046

Weir, A. A. S., Chappell, J., and Kacelnik, A. (2002). Shaping of hooks in New Caledonian crows. Science 297, 981-981. doi: 10.1126/science.1073433

Wild, J. M. (2015). "The avian somatosensory system: a comparative view," in Sturkie's Avian Physiology, ed. C. Scanes (Amsterdam: Elsevier), 55-69.
Wild, J. M., Arends, J. J., and Zeigler, H. P. (1985). Telencephalic connections of the trigeminal system in the pigeon (Columba livia): a trigeminal sensorimotor circuit. J. Comp. Neurol. 234, 441-464. doi: 10.1002/cne.9023 40404

Wild, J. M., and Farabaugh, S. M. (1996). Organization of afferent and efferent projections of the nucleus basalis prosencephali in a passerine, Taeniopygia guttata. J. Comp. Neurol. 365, 306-328.

Wild, J. M., and Zeigler, H. P. (1996). Central projections and somatotopic organisation of trigeminal primary afferents in pigeon (Columba livia). J. Comp. Neurol. 368, 136-152.

Williams, M. N., and Wild, J. M. (2001). Trigeminally innervated iron-containing structures in the beak of homing pigeons, and other birds. Brain Res. 889, 243-246. doi: 10.1016/s0006-8993(00)03114-0

Wiltschko, R., and Wiltschko, W. (1995). Magnetic Orientation in Animals. Berlin: Springer Verlag.

Wynn, J., Padget, O., Mouritsen, H., Morford, J., Jaggers, P., and Guilford, T. (2022). Magnetic stop signs signal a European songbird's arrival at the breeding site following migration. Science 375, 446-449. doi: 10.1126/science.abj4210

Zapka, M., Heyers, D., Hein, C. M., Engels, S., Schneider, N. L., Hans, J., et al. (2009). Visual but not trigeminal mediation of magnetic compass information in a migratory bird. Nature 461, 1274-1277. doi: 10.1038/nature0 8528

Ziswiler, V. (1965). Zur Kenntnis des Samenöffnens und der Struktur des hörnernen Gaumens bei körnerfressenden Oscines. J. Ornithol. 106, 785-793. doi: 10.1007/BF01670789

Zweers, G. A., Berkhoudt, H., and Berge, J. C. V. (1994). "Behavioral mechanisms of avian feeding," in Biomechanics of Feeding in Vertebrates, eds V. L. Bels, M. Chardon, and P. Vandewalle (Berlin: Springer), 241-279.

Conflict of Interest: The authors declare that the research was conducted in the absence of any commercial or financial relationships that could be construed as a potential conflict of interest.

Publisher's Note: All claims expressed in this article are solely those of the authors and do not necessarily represent those of their affiliated organizations, or those of the publisher, the editors and the reviewers. Any product that may be evaluated in this article, or claim that may be made by its manufacturer, is not guaranteed or endorsed by the publisher.

Copyright (c) 2022 Haase, Musielak, Warmuth-Moles, Leberecht, Zolotareva, Mouritsen and Heyers. This is an open-access article distributed under the terms of the Creative Commons Attribution License (CC BY). The use, distribution or reproduction in other forums is permitted, provided the original author(s) and the copyright owner(s) are credited and that the original publication in this journal is cited, in accordance with accepted academic practice. No use, distribution or reproduction is permitted which does not comply with these terms. 